\title{
Ventilator-Associated Pneumonia with Col-S Strains: A Successful Comeback of Colistin!
}

\author{
Mukhopadhyay C. ${ }^{1}$, Krishna S. ${ }^{1}$, Vandana K.E. ${ }^{1}$, Shenoy A. ${ }^{2}$ and Bairy I. $^{1}$ \\ ${ }^{1}$ Department of Microbiology and ${ }^{2}$ Department of Anesthesiology and MICU (Faculty in charge), Kasturba Medical College; Manipal, \\ Karnataka, India
}

\begin{abstract}
Emergence of multi and pan-drug resistant Gram-negative bacteria causing nosocomial infections in intensive care settings has become a challenge for clinicians. The mortality rate of ventilator-associated pneumonia (VAP) is known to increase when the initial microbiological diagnosis and antimicrobial therapy are inappropriate. We present a case of a 18-year-old man, who after being admitted following an accident, had developed VAP due to multidrug resistant Pseudomonas aeruginosa and Acinetobacter spp. and had a downhill clinical course despite broadspectrum antibiotic treatment. The strains were found to be Col-S, as the susceptibility was tested. Colistin was instituted, with remarkable recovery. It is imperative to diagnose VAP with multi-drug resistant strains as early as possible; colistin, the 'last resort' antibiotic, if instituted with proper monitoring at the right time, can be life saving.

Key-Words: Colistin, Col-S strain, ventilator-associated pneumonia.
\end{abstract}

Ventilator-associated pneumonia (VAP) is defined as the presence of new, persistent pulmonary infiltrates, not otherwise explained, appearing on chest radiographs, with at least two of the following criteria: temperature above $38^{\circ} \mathrm{C}$ or lower than $36^{\circ} \mathrm{C}$, leucocytosis or leucopenia (more than 10,000 or less than 4,000 cells $/ \mathrm{mm}^{3}$ ), and purulent respiratory secretions. It occurs more than 48-72 hours after mechanical ventilation and is judged not to have begun before an artificial airway was put in place [1]. In our intensive care unit, VAP is the most common hospital-acquired infection associated with mechanical ventilation, with an incidence of $45.4 \%$ [2].

This disease is a challenge for clinicians, due to varied clinical presentations of the patients as well as for microbiologists, who need to confirm bacteriological diagnosis as early as possible [3]. A universally-acceptable approach to diagnose and manage a case of VAP is still not available and remains a subject of investigation because a gold standard for diagnosis of VAP has never been clearly established [4]. Although lung biopsy, bronchoalveolar lavage (BAL), protected - specimen brush (PSB), and miniBAL are regarded as the best methods for the diagnosis of VAP, with high sensitivity and specificity, various studies have also demonstrated the efficacy of quantitative culture of endotracheal aspirate (ETA) as a diagnostic method in intubated patients [5]. The isolation of multiple drug resistant (MDR) Acinetobacter spp. and Pseudomonas aeruginosa is an increasingly common phenomenon observed in various intensive care units [6-9]. In recent years, strains of Acinetobacter spp. and $P$. aeruginosa nonsusceptible to nearly all classes of antibiotics, including

\section{Received on 31 May 2008; revised 19 October 2008.}

Address for correspondence: Dr. . Chiranjay Mukhopadhyay. Associate Professor, Department of Microbiology, Kasturba Medical college, Manipal-576104, Karnataka, India; Phone: 91-820-2571201(office), Extn: 22322, 91-9845513057(mobile) Fax: 91-820-2571927; E-mail: chiranjay@yahoo.co.in.

The Brazilian Journal of Infectious Diseases

2008;12(5):444-446. (C) 2008 by The Brazilian Journal of Infectious Diseases and Contexto Publishing. All rights reserved. carbapenems, one of the most effective antimicrobials for these pathogens $[8,10]$, have been identified more frequently as the VAP pathogens $[7,8,11]$. Colistin, a polymyxin antibiotic appears as an appropriate therapeutic alternative. The amount of data available regarding epidemiological and clinical characteristics of VAP, due to Gram-negative bacilli susceptible only to colistin (Col-S), is limited [12,13]. We present the first case of VAP from India caused by multi-drug resistant Col-S strain, where the use of colistin subsequently changed the outcome of the patient.

\section{Case Report}

In December 2007, an 18-year-old coolie, with no co-morbid illness was admitted to our multidisciplinary intensive care unit (MICU), with a history of a cement slab that fell on his chest and abdomen. The diagnosis at admission was trauma with hemoperitoneum, hemothorax, pelvic hematoma, splenic gastro-esophageal tear, left hip and vertebral fracture, with paraplegia. The patient was in hypovolemic shock, with massive bleeding, multiple abrasions all over the body, decreased breathing sounds, tachycardia, tachypnea, and hypotension, with a Glasgow Coma Scale (GCS) of 10. He was revived with emergency fluid infusions, blood transfusions and laparotomy. Sixteen samples, including culture of the blood, ETA, tracheal aspirate (TA), pleural fluid, peritoneal fluid, throat swab, urine, decubitus ulcer swab and serology for HIV, HbsAg and widal were sent to microbiology. ETAs were collected on days 1 and 4 of intubation, and the four TAs were collected thereafter. The sample collected on day 4 was grossly muco-purulent, thick, yellowish, foul-smelling and large in volume. Gram staining revealed numerous pus cells and two types of Gram-negative bacilli. On culture, the sample showed heavy growth $\left(>10^{5} \mathrm{cfu} / \mathrm{mL}\right)$ of $P$. aeruginosa (Figure 1) and Acinetobacter spp., which were resistant to antipseudomonal penicillin, third generation cephalosporin, aminoglycosides, quinolones, monobactum and carbapenems. 
A diagnosis of early onset VAP was made, with a clear zone of homogenous opacity forming in the lower lobe of the right lung on chest X-ray at the end of the first week. The patient was started empirically on cefoperazone-sulbactum (1g bd i.v.) and netilmicin (100 mg bd i.v.) from day 1, and as his clinical condition did not improve, piperacillin-tazobactum replaced cefoperazone-sulbactum during the second week. Tracheostomy was done on day 5 and TAs on days 7, 10, 15 yielded equal quantitative growth, with similar antibiograms, consistently. With no clinical improvement and worsening pleural effusion, the antibiotic was again changed, this time to meropenem $1 \mathrm{~g}$ bd i.v. during the third week. Pleural fluid grew the same two strains on day 20, and blood culture reported the same strain of $P$. aeruginosa on day 22. Fever continued to spike, with no clinical improvement, leaving little choice to the treating clinicians.

At this stage, colistin was tested in vitro; both strains were sensitive to it. The patient was immediately started on colistin (1.5 mg/kg every eight hours), which was continued for the next 14 days, along with extensive CT-guided drainage of pyopneumothorax. He made a remarkable clinical recovery on day 5 of colistin treatment. Fever began touching base line, with the chest X-ray showing breakdown of opacities (Figures 2 and 3). Meropenem was continued, along with colistin, for the next two weeks. A final TA collected on day 30 was sterile, and lung fields were clear on auscultation. He showed no signs of neuro or nephrotoxicity. The patient had a prolonged recovery, with a duration of over 70 days in the hospital, including 40 days in the intensive care unit, with ventilatory and tracheostomy support. The long duration of hospital stay, repeated change of antibiotics and prolonged immobilization, with disuse atrophy of the limbs, resulted in bedsores, nutritional insufficiency and paraplegia, which contributed to the current morbidity. He is bed-ridden in a

Figure 1. Growth of multi-drug resistant Pseudomonas aeruginosa from endotracheal aspirate on nutrient agar.

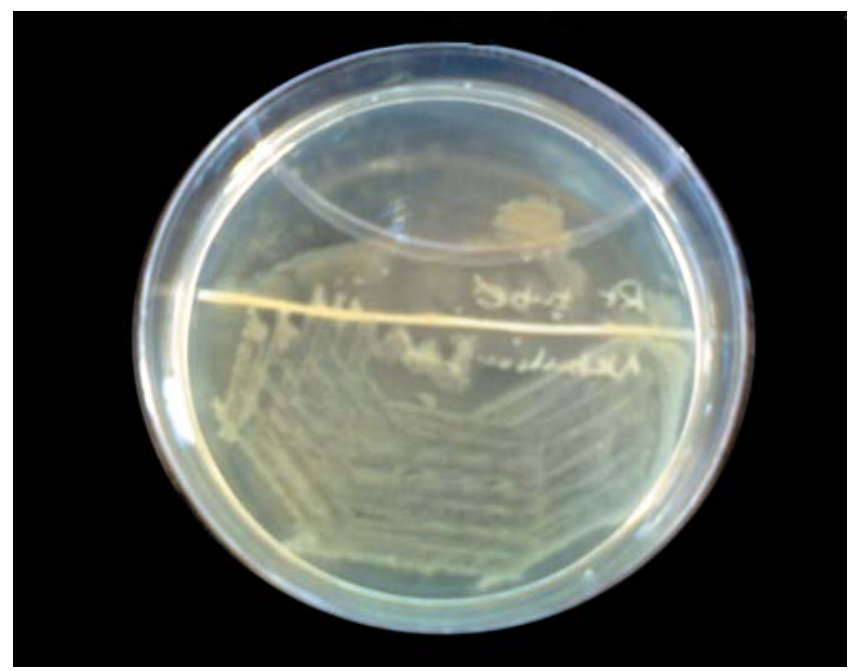

local nursing home, with elastic-crape bound legs, spinal braces and a gastrojejunostomy feeding tube to date.

\section{Discussion}

This was a typical case of early-onset VAP on day 3, with a Clinical Pulmonary Infection Score (CPIS) score of 9. The patient's ETA/TA samples grew two MDR organisms - $P$. aeruginosa and Acinetobacter sp., with no clinical response to a broad range of antibiotics (cefoperazone - sulbactam, piperacillin-tazobactum, and meropenem). It is known that VAP episodes caused by the so-called 'high-risk pathogens', particularly the nonfermenting Gram-negative bacilli, $P$. aeruginosa and Acinetobacter spp., are associated with high mortality rate, with a relative risk of 2.5 when compared with other microorganisms $[14,15]$. The incidence of nosocomial infections due to MDR pathogens has grown impressively during recent decades; they show resistance to imipenem and meropenem, the most effective antimicrobials of the last decade. Several factors that are related to the acquisition of VAP due to MDR microorganisms have been detected, including, especially, $>7$ days duration of mechanical ventilation, prior antimicrobial therapy and use of broadspectrum antibiotics [16]. In our case, the patient developed VAP only on day 4 in the MICU set up, which signifies that a more cautious approach and preventive measures must be taken against environmental stress in artificially-ventilated patients.

Colistin, which was widely used in 1950s, was discontinued from parenteral clinical usage in the 1970s, because of its severe adverse effects of nephrotoxicity and neurotoxicity. Moreover, the clinical experience with this antibiotic is poor [14]. Although there are recommendations to begin colistin as empirical antimicrobial therapy in VAP [14], we consider it as inappropriate to use it as 'first line therapy'. Physicians should

Figure 2. Chest radiograph before colistin treatment.

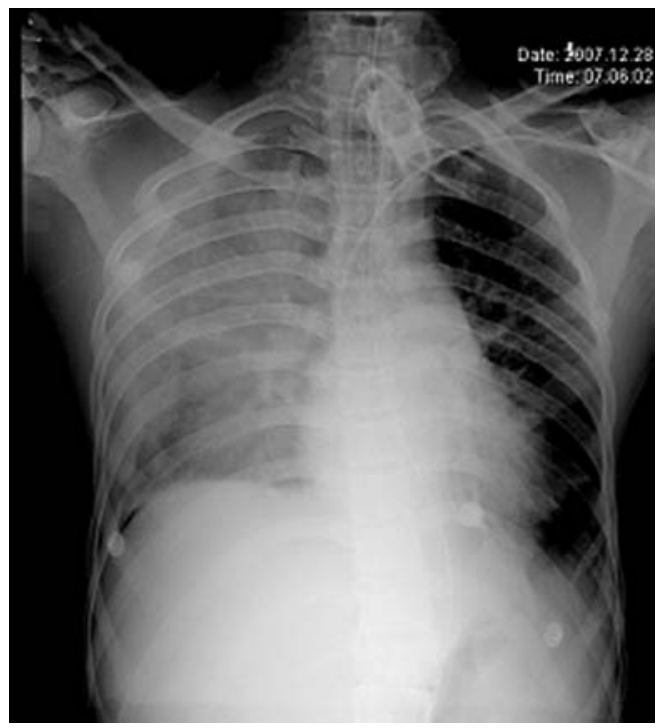


Figure 3. Chest radiograph after colistin treatment.

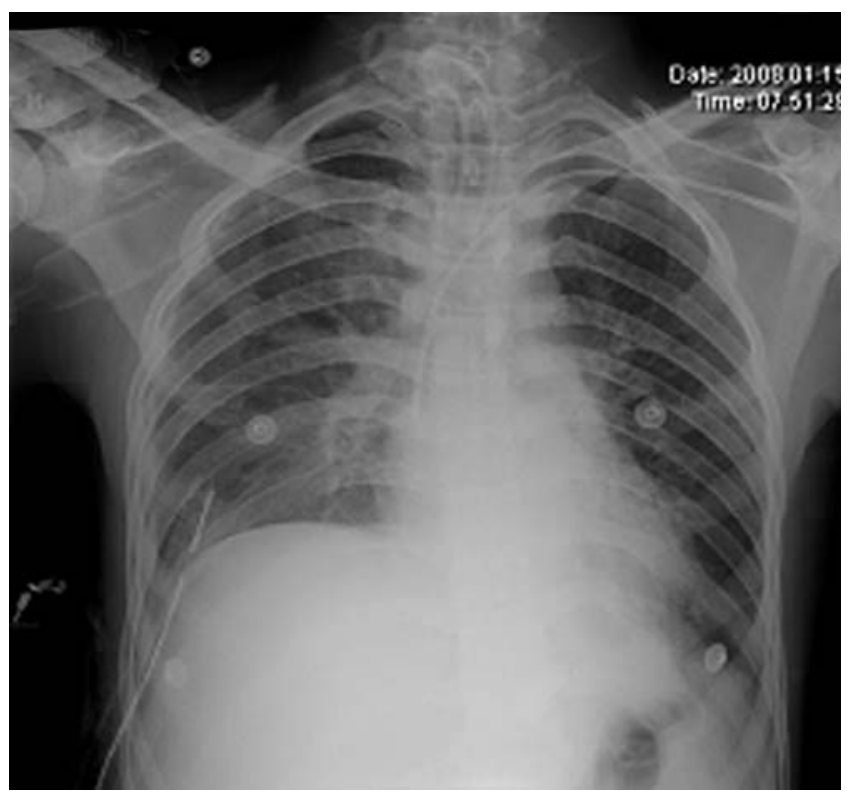

know their own environment, and microbiologists should consider colistin as an option when there are risk factors for MDR isolates. It is always a priority to identify the colistin susceptibility of MDR Acinetobacter spp. and Pseudomonas aeruginosa strains; such information will assist in choosing the appropriate antibiotic. Delay in the initiation of antimicrobial therapy increases the VAP mortality rate $[17,18]$. A higher mortality rate has been observed in patients who did not receive appropriate therapy or received it after a delay, in comparison to those who received appropriate therapy. This was one of the few cases of timely testing of susceptibility and successful institution of colistin for the treatment of VAP, and it is probably the first from India.

\section{Conclusion}

With the emergence of multi and pan drug-resistant bacterial strains, particularly in intensive care units, today's medical world is now realizing the limitations of antibiotics. This case highlights the importance of in vitro testing of colistin in the clinical lab in cases of multi and pan-drug resistant organisms. Parentral colistin can be judiciously considered as a dependable option to achieve a satisfactory clinical response in the treatment of VAP, especially to treat Col-S strains of $P$. aeruginosa and Acinetobacter spp.

\section{Acknowledgements}

We acknowledge the utmost attention, care and management by Dr. (Prof.) George Varghese (Department of Medicine, Unit IV), Dr. (Prof.) Sampath Kumar and other doctors from the Departments of Surgery (unit II) and Anesthesiology, which led to the successful recovery of the patient. We also acknowledge the cooperativeness on behalf of the relatives of the patient.

\section{References}

1. Rello J., Artur J., Baraibar J., et al. International conference for the development of consensus on the diagnosis and treatment of ventilator associated pneumonia. Chest 2001;120: 955-70.

2. Dey A., Bairy I. Incidence of multidrug-resistant organisms causing ventilator-associated pneumonia in a tertiary care hospital: A nine months' prospective study Ann Thorac Med 2007; 2: 52-7.

3. Cook D., Mandel L. Endotracheal Aspiration in diagnosis of VAP. Chest 2000; 117: 195-7.

4. Chastre J., Fagon J.Y. Ventilator associated pneumonia. Am J Respir Crit Care Med 2002;163:867-903.

5. Bergmans D.C., Bonten M.J., De Leeuw P.W., Stobberingh E.E. Reproducibility of quantitative cultures of endotracheal aspirates from mechanically ventilated patients. J Clin Microbiol 1997;35:796-8.

6. Crouch Brewer S., Wunderink R.G., Jones C.B., Leeper K.V. Jr. Ventilator-associated pneumonia due to Pseudomonas aeruginosa. Chest 1996;109:1019-29.

7. Rahal J.J., Urban C. Acinetobacter. Semin Respir Crit Care Med 2000;21:341-8.

8. Gales A.C., Jones R.N., Forward K.R., et al. Emerging importance of multi-drug resistant Acinetobacter species and Stenotrophomonas maltophilia as pathogens in seriously ill patients: geographic patterns, epidemiological features, and trends in the SENTRY Antimicrobial Surveillance Program (1997-1999). Clin Infect Dis 2001;32:S104-S13.

9. Mukhopadhyay C., Bhargava A., Ayyagari A. Role of mechanical ventilation \& development of multidrug resistant organisms in hospital acquired pneumonia. Indian J Med Res 2003;118:229-35.

10. Gales A.C., Jones R.N., Turnidge J., et al. Characterization of Pseudomonas aeruginosa isolates: occurrence rates, antimicrobial susceptibility patterns, and molecular typing in the global SENTRY Antimicrobial Surveillance Program, 1997-1999. Clin Infect Dis 2001;32:S146-S55.

11. Rý'F., Luna C.M., Menga G., et al. Análisis de neumonýa asociada a ventilación mecánica en 6 hospitales de Buenos Aires. [Analysis of ventilator-associated pneumonia in 6 hospitals of Buenos Aires.] 13th Argentinian Congress of Intensive Therapy. September 2002, Buenos Aires, Argentina.

12. Garnacho-Montero J., Ortiz-Leyba C., Jiménez-Jiménez F.J., et al. Treatment of multi-drug-resistant Acinetobacter baumanni VAP with intravenous colistin: a comparison with imipenamsusceptible VAP. Clin Infect Dis 2003;36:1111-8.

13. Levin A.S., Barone A.A., Penco J., et al. Intravenous colistin as therapy for nosocomial infections caused by multi-drug-resistant Pseudomonas aeruginosa and Acinetobacter baumanni. Clin Infec Dis 1999;28:1008-11.

14. Rios F.G., Luna C.M., Maskin B., et al. Ventilator-associated pneumonia due to colistin susceptible-only microorganisms. Eur Respir J 2007;30:307-13.

15. Chastre J., Fagon J.Y. Pneumonia in the ventilator-dependent patient. In: Tobin M.J., ed. Principles and Practice of Mechanical Ventilation. New York, McGraw-Hill Inc., 1994; pp. 857-90.

16. Trouillet J.L., Chastre J., Vuagnat A., et al. Ventilator associated pneumonia caused by potentially drug-resistant bacteria. Am J Respir Crit Care Med 1998;157:531-9.

17. Le Moyec L., Racine S., Le Toumelin P., et al. Aminoglycoside and glycopeptide renal toxicity in intensive care patients studied by proton magnetic resonance spectroscopy of urine. Crit Care Med 2002;30:1242-5.

18. Chastre J., Fagon J.Y. Ventilator-associated pneumonia. Am J Respir Crit Care Med 2002;165:867-903. 